\title{
Ragni di Grecia VI. Specie nuove o interessanti delle isole Ionie e della Morea $(\text { Araneae })^{1}$
}

di

\section{Paolo Marcello BRIGNOLI}

Con 21 figure nel testo

Nel corso delle ricerche in Grecia svolte da vari anni da alcuni studiosi del Museo di Storia Naturale di Ginevra (B. Hauser, I. Löbl, V. Mahnert) è stato raccolto un buon numero di ragni anche cavernicoli ed endogei di elevato interesse. In questa nota pubblico i primi risultati dello studio di questo materiale affidatomi, con la consueta gentilezza, dal Dr. Bernd Hauser (Laboratorio degli Artropodi e degli Insetti inferiori) che vivamente ringrazio.

Per ragioni di tempo mi sono limitato ad esaminare solo il materiale relativo ad un certo numero di famiglie o generi di cui ho attualmente in istudio anche raccolte di altre provenienze.

Salvo contraria indicazione, tutto il materiale qui trattato è conservato nelle collezioni del Muséum d'Histoire naturelle de Genève.

\section{Fam. OONOPIDAE}

\section{Dysderina loricatula Roewer, 1942}

Leucade - Bosco di ulivi sopra Kaligoni (vagliatura presso la riva di un fiume sotto Quercus ilex ed ulivi), 26.III.71, B. Hauser leg., 1 \% (Gr-71/26).

${ }^{1}$ Per i miei cinque precedenti lavori sulla Grecia, vedi: Senckenberg. biol. 49: 259-64 (1968), Fragm. ent. 7: 231-41, Mitt. Zool. Mus. Berl. 47: 255-67 (1971), Senckenberg. biol. 53: 287-89, Revue suisse Zool. 79: 861-69 (1972). 
Leucade - Sopra Phryni (vagliatura sotto Pinus), 27.III.71, I. Löbl leg., 1 ㅇ (Gr-71/39).

Corfù - $3 \mathrm{~km}$ a Ovest di Kassiopi (vagliatura sopra un ruscello asciutto sotto

Laurus), 12.IV.72, B. Hauser leg., 1 ㅇ (Io-72/28).

Corfù - Presso la grotta Peristerograva, Lutses, 490 m, 12.IV.72, V. Mahnert

leg., 1 ô, 1 ㅇ (Io-72/31).

Itaca - Anoghi, $500 \mathrm{~m}$ (vagliatura sotto Quercus), 19.IV.72, B. Hauser leg.,

1 우 (Io-72/55A).

Uno degli Oonopidae europei meno rari; nota di Grecia dell'Eubea (SIMON, 1884 c), di Olimpia nel Peloponneso (Giltay, 1932) e dell'Attica (HadjISSARANTOS, 1940).

\section{Oonops mahnerti n. sp.}

Leucade - Karya (bosco di ulivi sopra il villaggio), 28.III.71, V. Mahnert leg.,

1 ㅇ (Holotypus: Gr-71/44).

Descrizione - + ( $\widehat{o}$ ignoto): prosoma giallo citrino, senza peculiarità; occhi nella normale disposizione, assai simili come dimensioni (LA: MP: LP = $7: 6: 5$ ). Labium piccolo, triangolare, tanto lungo che largo; sterno cordiforme, liscio, gialliccio, terminante con una piccola punta ottusa separante le IV coxe all'incirca del loro diametro. Cheliceri e palpi senza peculiarità. Zampe di color giallo pallido, femori I con 2 spine prolaterali; tibie I-II con 6-6 spine; metatarsi I-II con 2-2 spine. Opistosoma bianco, villoso; vulva, v. fig. 2. Dimensioni (in $\mathrm{mm}$, leggermente approssimate): prosoma lungo 0,77 , largo 0,60 ; opistosoma lungo 1,12 . Lunghezza totale: 1,89 .

\begin{tabular}{|c|c|c|c|c|c|c|}
\hline Zampe & Femore & Patella & Tibia & Metatarso & Tarso & Totale \\
\hline I & 0,62 & 0,27 & 0,78 & 0,47 & 0,25 & 2,39 \\
II & 0,57 & 0,25 & 0,62 & 0,47 & 0,23 & 2,14 \\
III & 0,52 & 0,25 & 0,47 & 0,47 & 0,21 & 1,92 \\
IV & 0,93 & 0,27 & 0,77 & 0,75 & 0,23 & 2,95 \\
\hline
\end{tabular}

Derivatio nominis: ho il piacere di dedicare questa specie al suo raccoglitore, Volker Mahnert.

Affinità: quasi tutte le specie paleartiche di Oonops sono insufficientemente note, visto che la parziale revisione del DE DALMAS (1916), unico studio d'insieme su questo gruppo, permette solo una distinzione sulla base di caratteri quanto mai superficiali. Nonostante ciò, grazie ad essa è possibile stabilire che questa femmina delle Isole Ionie non è ascrivibile a nessuna delle specie note. La sua chetotassi infatti è assai caratteristica e si può avvicinare entro certo limiti solo 


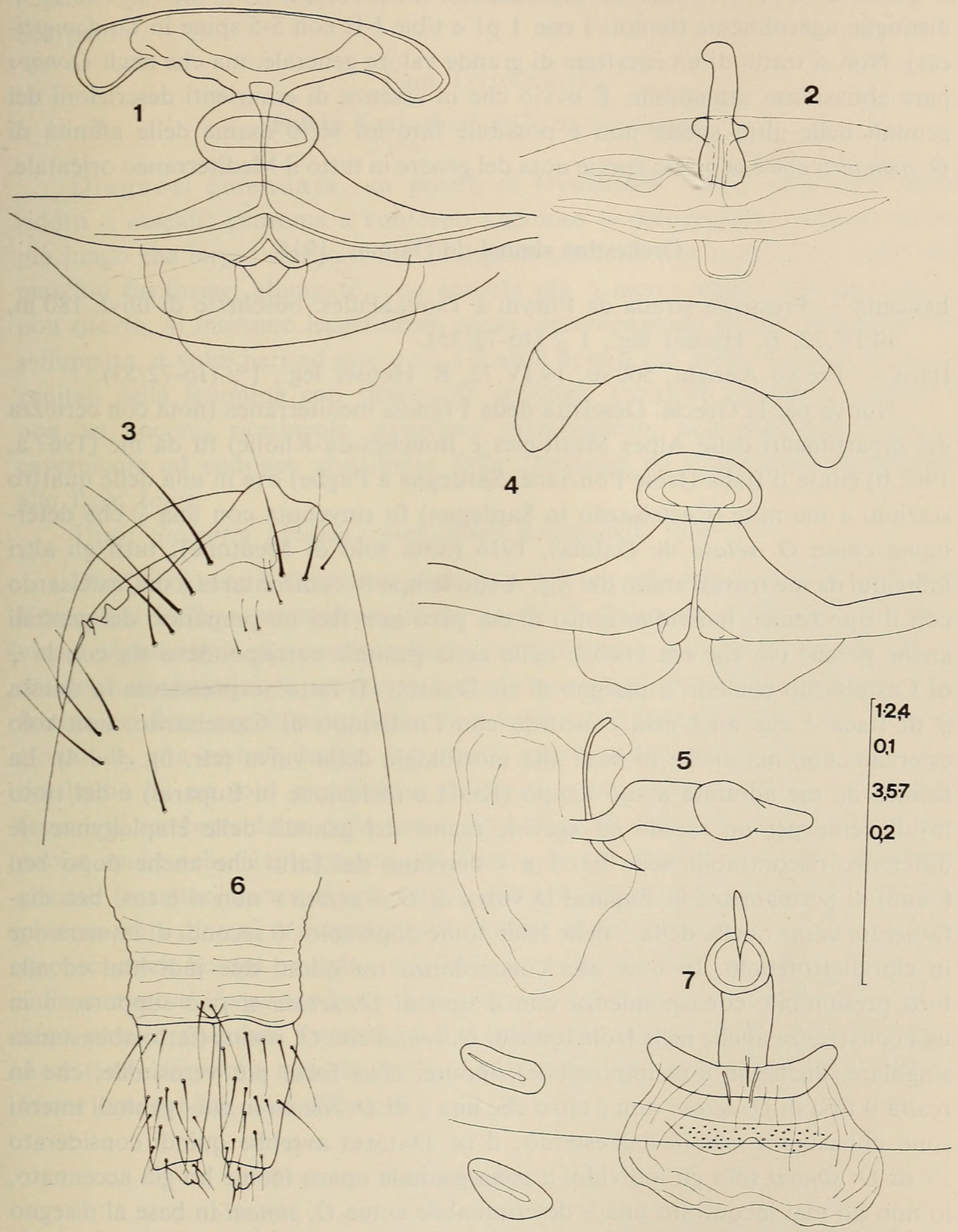

FIG. 1-7.

Orchestina simoni de Dalmas, 1916 - Fig. 1: vulva; Fig. 6: filiere e colulo. Orchestina « setosa ", de Dalmas 1916 - Fig. 4: vulva (individuo di Castelsardo, Sardegna). Oonops mahnerti n. sp. Fig. 2: vulva. Folkia lugens n. sp. - Fig. 3: chelicero; Fig. 5, 7: vulva di lato e dal basso. 
a quella di $O$. domesticus de Dalmas, 1916, da cui però $O$. mahnerti n.sp. si distingue agevolmente (femori I con $1 \mathrm{p} 1$ e tibie I-II con 5-5 spine in $O$. domesticus). Non si tratta di un carattere di grande valore generale, ma che negli Oonops pare abbastanza attendibile. È ovvio che in assenza di esaurienti descrizioni dei genitali delle altre specie non è possibile fare un serio esame delle affinità di $O$. mahnerti che è la prima specie nota del genere in tutto il Mediterraneo orientale.

Orchestina simoni de Dalmas, 1916

Leucade - Presso la strada da Phryni a-Tsoukalades, boschetto di ulivi, $180 \mathrm{~m}$, 14.IV.72, B. Hauser leg., 1 ô (Io-72/35).

Itaca - Presso Anoghi, 500 m, 19.IV.72, B. Hauser leg., 1 (Io-72/53).

Nuova per la Grecia. Descritta della Francia mediterranea (nota con certezza dei dipartimenti delle Alpes Maritimes e Bouches-du-Rhône) fu da me (1967 a, 1967 b) citata d'Italia (Isole Ponziane, Sardegna e Puglie) ove in una delle quattro stazioni a me note (Castelsardo in Sardegna) fu rinvenuta con una $q$ che determinai come $O$. setosa de Dalmas, 1916 (nota solo di Mentone); tutti gli altri

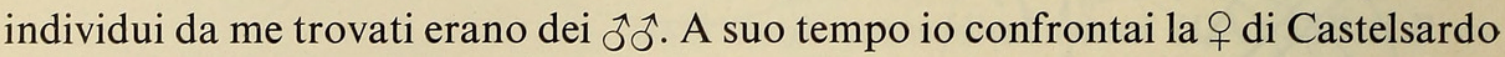
con il tipo (unico individuo noto) di cui però non feci un preparato dei genitali anche perchè ciò che era visibile nella zona genitale corrispondeva sia con la $q$ di Castelsardo che con il disegno di DE DALMAS. Il fatto sorprendente in questa O di Itaca è che anch'essa concorda con l'individuo di Castelsardo, non solo esternamente, ma anche in base alla morfologia della vulva (cfr. fig. 1 e 4). La tecnica da me adottata a suo tempo ( $\mathrm{KOH}$ e inclusione in Euparal) è del tutto insufficiente per un rapido ed agevole esame dei genitali delle Haplogynae; le differenze riscontrabili nelle fig. 1 e 4 derivano dal fatto che anche dopo ben 6 anni di permanenza in Euparal la vulva di $O$. " setosa » non si è così ben diafanizzata come quella della $q$ delle Isole Ionie dopo solo 30 secondi di immersione in clorallattofenolo. In base alla concordanza tra questi due individui ed alla loro presumibile corrispondenza con il tipo di $O$. setosa si può supporre o in una convivenza anche nelle Isole Ionie di $O$. setosa con $O$. simoni (fatto abbastanza singolare, anche se non impossibile) oppure, cosa forse più verosimile, che in realtà il tipo di $O$. setosa non è altro che una $q$ di $O$. simoni in cui i genitali interni sono abbastanza visibili dall'esterno; il DE DALMAS avrebbe quindi considerato 우 di $O$. simoni solo gli individui a zona genitale opaca (come ho già accennato, io non ho mai incontrato una 9 determinabile come $O$. simoni in base al disegno di DE DALmAS). Spero di poter risolvere tra breve questo problema confrontando of tipiche di $O$. simoni e $O$. setosa (in una eventuale sinonimia $O$. simoni ha priorità di pagina).

Con O. simoni gli Oonopidae noti di Grecia divengono 5; oltre alle specie qui ricordate infatti HadjisSARAntos (1940) citò dell'Attica Opopaea punctata 
(Cambridge, 1872) ed io (1972) ho recentemente descritto Orchestina ebriola di Scarpanto.

Fam. DYSDERIDAE

Folkia Kratochvil, 1970 nuovo status

Diagnosi emendata: un genere di Dysderidae stricto sensu con occhi ridotti o assenti; prosoma a contorno angoloso («poligonale »); labium molto più lungo che largo; sterno ovale allungato; cheliceri non modificati; bulbo del maschio fusiforme, allungato, con embolo più o meno lungo, robusto, sottile, non ritorto, al massimo leggermente curvo, accompagnato da un conductor ben sviluppato, a volte pari ad esso e da 1-2 altre apofisi più brevi, ma ben evidenti; genitali della femmina con ricettacolo seminale anteriore semplice, allungato, non ad ancora, ricettacolo posteriore membranoso, non sclerificato. Specie cavernicole od endogee, distribuite dalla Dalmazia ed Erzegovina meridionali alle Isole Ionie.

Tipo: Stalagtia (Folkia) purkrabeki Kratochvil, 1970.

Discussione: Kratochvil descrisse Folkia come sottogenere di Stalagtia Kratochvil 1970, comprendente poche specie di Dalmazia meridionale, Erzegovina e Montenegro. A parte le indubbie somiglianze di habitus tra le varie specie dei due sottogeneri è da notare però che la morfologia del bulbo è alquanto differente nei due gruppi; nelle Stalagtia propriamente dette il bulbo è più o meno sferoidale, con embolo lungo, fortemente curvo, quasi ritorto rispetto al corpo del bulbo ed un solo piccolissimo conductor, non sempre presente. I genitali femminili sembrerebbero dello stesso tipo di quelli delle Folkia. Giacchè a mio parere anche nelle Haplogynae è opportuno dare il massimo peso alla forma dei genitali (caratteri apomorfi) rispetto a generali somiglianze di habitus (caratteri plesiomorfi o dovuti a convergenze) sarebbe stato possibile o eliminare Folkia oppure, come ho qui preferito, scinderlo da Stalagtia. Solo maggiori conoscenze sulle numerose Harpactea balcaniche permetteranno di chiarire se sia più opportuno, come ora sembra, il metodo dello « splitting » oppure quello del « lumping ». Accettando il primo metodo ritengo impossibile mantenere nello stesso genere forme con genitali così differenti.

Folkia lugens n. sp.

Leucade - Presso il bivio per Sivota, $140 \mathrm{~m}$ (campione di suolo sotto Quercus; selettore Berlese), 16.IV.72, B. Hauser leg., 1 ㅇ (Holotypus); Io-72/45).

Descrizione - 우 ( $\widehat{0}$ ignoto): prosoma a contorno lievemente angoloso, gialliccio, basso, senza tracce di fovee; occhi assenti, salvo gli anteriori (LA) depigmentati. Labium più lungo che largo; sterno ovale allungato con punta 
ottusa, separante le IV coxe all'incirca del loro diametro. Cheliceri, v. fig. 3; palpi senza peculiarità. Femori I con 3 spine pl. Opistosoma bianchiccio, ovale, allungato; vulva, v. fig. 5, 7 .

Dimensioni (in $\mathrm{mm}$, leggermente approssimate); prosoma lungo 0,95 , largo 0,67; opistosoma lungo 1,70. Lunghezza totale: 2,65.

\begin{tabular}{|c|c|c|c|c|c|c|}
\hline Zampe & Femore & Patella & Tibia & Metatarso & Tarso & Totale \\
\hline I & 0,75 & 0.43 & 0,65 & 0,55 & 0,25 & 2,63 \\
II & 0,65 & 0,41 & 0,50 & 0,47 & 0,25 & 2,28 \\
III & 0,52 & 0,31 & 0,37 & 0,46 & 0,25 & 1,91 \\
IV & 0,72 & 0,41 & 0,56 & 0,69 & 0,25 & 2,63 \\
\hline
\end{tabular}

Derivatio nominis: il participio latino « lugens » equivale a « piangente ».

Discussione e affinità: la sistematica a livello generico nei Dysderidae (come spesso nelle Haplogynae) si è basata in passato essenzialmente su caratteri di valore notoriamente incerto, quali la posizione degli occhi, la forma del prosoma o la chetotassi delle zampe, tenendo in poco conto i genitali maschili ed ignorando quelli femminili. Il risultato di questa situazione è che molto spesso è arduo decidere sulla posizione generica di una specie di cui sia noto solo un sesso ed in particolare quando questo è il femminile. Come primo passo per decidere sulla posizione di questa forma microftalma ho ammesso come ipotesi di lavoro l'esistenza di stretti rapporti fra tutte le specie correntemente ascritte ai generi Stalagtia Kratochvil, 1970, Folkia Kratochvil, 1970 e Minotauria Kulczynski, 1903 interpretando d'altro canto questo complesso di forme più o meno cavernicole come delle Harpactea lato sensu specializzate. Questi due complessi (concordo pienamente con AlicAta nel considerare Harpactea un genere non omogeneo) raggrupperebbero in pratica gran parte dei piccoli Dysderidae mediterranei detriticoli o cavernicoli con bulbo relativamente semplice ad embolo più o meno nettamente staccato dal corpo del bulbo e di solito con un conductor ben evidente.

Ammesso l'inserimento in questa serie filetica di questa $q$ delle Isole Ionie per l'insieme dei caratteri morfologici restava il problema del genere a cui attribuirla, problema quasi insolubile in assenza del $\hat{\jmath}$. Ho deciso di attribuire questa specie a Folkia più che altro per una certa somiglianza dei suoi genitali con quelli di F. purkrabeki Kratochvil, 1970 e F. haasi (Reimoser, 1929) nonchè per la riduzione oculare (fenomeno che però nulla dice sia esclusivo del gruppo Stalagtia-Folkia-Minotauria).

$F$. lugens n.sp. è agevolmente distinguibile dalle altre specie di questo gruppo di cui sono noti i genitali delle 
Stalagtia hercegovinensis (Nosek, 1905), S. monospina (Absolon \& Kratochvil, 1933), S. krivosijana Kratochvil, 1970 e S. skadarensis Kratochvil, 1970 (per tutte, cfr. KratochVIL, 1970); delle altre specie, S. inermis (Absolon \& Kratochvil, 1933), S. folki Kratochvil, 1970, e S. mrazeki (Nosek, 1903), sono ben differenti per dimensioni (maggiori) e chetotassi (più spine).

Solo S. pauciaculeata (Fage, 1943), descritta come "varietà » e considerata da KRAtochvil buona specie si può avvicinare per chetotassi a F. lugens n.sp. (femori I con solo $5 \mathrm{pl}$ ), ma è di più grandi dimensioni (lunghezza $4,5 \mathrm{~mm}$ ). Considerarla affine a F. lugens n.sp. è del tutto prematuro. Quanto alle «Folkia» di Creta, v. più avanti.

Per scrupolo ho paragonato la nuova specie anche con tutte le Harpactea (ed Harpactocrates) dei Balcani, senza però giungere a nessun risultato degno di nota.

\section{Le « Folkia » di Creta}

Nel 1928 Roewer descrisse Stalita cretica su di una $q$ della grotta di Topolia; nel 1945 FAGE citò la stessa specie della grotta di Melidhoni, descrivendone per la prima volta il maschio e notando alcune differenze tra la chetotassi del materiale a sua disposizione e quella riportata da RoEwER. KRATOCHVIL (1970), basandosi su questi dati e su di una femmina di Melidhoni propose di considerare queste due popolazioni come due sottospecie distinte, trasferendole nello stesso tempo a Stalagtia (Folkia). KRATOCHVIL apparentemente non rilevò un fatto già osservato da FAGE e cioè l'estrema somiglianza tra il bulbo di « Stalita » cretica e quello di Minotauria attemsi Kulczynski, 1903, della grotta del Labirinto, una specie microftalma (tipo ed unica del genere) la cui chetotassi non è molto diversa da quella delle forme di RoEwer e FAGE.

Per quale motivo FAGE non abbia trasferito a Minotauria la specie di RoEwER mi è oscuro; in base alle descrizioni ritengo non possano sussistere dubbi sul fatto che queste forme sono tra loro strettamente affini e che si debba quindi scrivere:

Minotauria cretica cretica (Roewer, 1928) comb. nov. e

M. cretica fagei (Kratochvil, 1970) comb. nov.

Come carattere distintivo tra Minotauria Kulczynski e Folkia Kratochvil considero la forma del bulbo, assai semplice nelle Minotauria, con embolo assai breve e privo di conductor; a questo riguardo il rapporto tra Folkia e Minotauria sembra simile a quello esistente tra Parachtes Alicata, 1964 e Harpactea Bristowe, 1939.

Tenendo conto della « accuratezza » delle descrizioni di ROEWER si potrebbe anche supporre una sinonimia tra $M$. attemsi e $M$. cretica, visto però che le due 
specie sono di grotte differenti, ciò non è sicuro; nulla esclude per di più che a Creta convivano parecchie Minotauria più o meno adattate alla vita cavernicola.

\section{Harpactea loebli n. sp.}

Cefalonia - Sul margine della strada presso Livadion, 1.IV.71, I. Löbl leg., 1 ơ (Holotypus; Gr-71/65).

Descrizione - $\widehat{o}(q$ ignota; N.B. v. poi): prosoma bruniccio, finemente punteggiato, basso, a contorno leggermente poligonale; occhi in un gruppo compatto, posteriori in una linea a debole concavità anteriore, poco diversi tra loro (LA/LP : MP = 7:6); LA accostati tra loro, come pure i posteriori. Cheliceri, rossicci, robusti, esternamente granulosi, con $2+2$ denti. Palpo, v. fig. 8-10. Labium quadrangolare, molto più lungo che largo. Sterno ovale allungato, finemente reticolato, bruniccio; termina con una piccola punta ottusa separante le IV coxe meno del loro diametro. Zampe giallicce, I-II più robuste con coxe allungate; femori I con 2 spine pl, tibie II con 2 pl. Opistosoma sottile, allungato, bianchiccio. Dimensioni (in $\mathrm{mm}$, leggermente approssimate): prosoma lungo 1,85, largo 1,20; opistosoma lungo 1,70. Lunghezza totale: 3,55.

\begin{tabular}{|c|c|c|c|c|c|c|}
\hline Zampe & Femore & Patella & Tibia & Metatarso & Tarso & Totale \\
\hline I & 1,50 & 1,00 & 1,50 & 1,45 & 0,50 & 5,95 \\
II & 1,37 & 0,82 & 1,20 & 1,20 & 0,45 & 5,04 \\
III & 1,05 & 0,45 & 0,67 & 0,90 & 0,35 & 3,42 \\
IV & 1,37 & 0,62 & 1,00 & 1,37 & 0,35 & 4,71 \\
\hline
\end{tabular}

Derivatio nominis: dedico con piacere questa specie al suo raccoglitore, Ivan Löbl.

Affinità: l'unico studio d'insieme recente sul genere Harpactea è quello limitato alle specie italiane di AlicAta (1966 a). Come già accennato, sono pienamente d'accordo con l'amico di Catania nel giudicarlo quanto mai eterogeneo e da frazionare. È però da tener presente che, a parte le troppe specie balcaniche poco note, ve ne sono probabilmente ancora non poche da descrivere (ne ho per es. varie del Vicino Oriente) e che quindi è opportuna molta prudenza nella delimitazione di gruppi di specie (e generi).

Della penisola balcanica sono state descritte non poche Harpactea; di queste alcune sono state trasferite - a ragione - da Alicata (1966 b) a Dasumia (H. amoena (Kulczynski), H. chyzeri (Kulczynski), H. kusceri Kratochvil). Delle altre alcune hanno un bulbo a terminazione più o meno complessa, ma sempre molto di più di $H$. loebli n.sp., così H. babori (Nosek, 1905), H. henschi (Kul- 
czynski, 1915), H. saeva (Herman, 1879). Quanto ad H. abantia (Simon, 1884), questa specie sembra del gruppo corticalis (cfr. HADJISSARANTOS, 1940), mentre H. krueperi (Simon, 1884) di cui non esistono figure, è anch'essa con bulbo semplice, ma di altro tipo (" bulbo... stylo terminali sat longo gracillimo simplice, fere recto atque ad apicem paulo hamato", Simon, op. cit.: 345). H. sturanyi

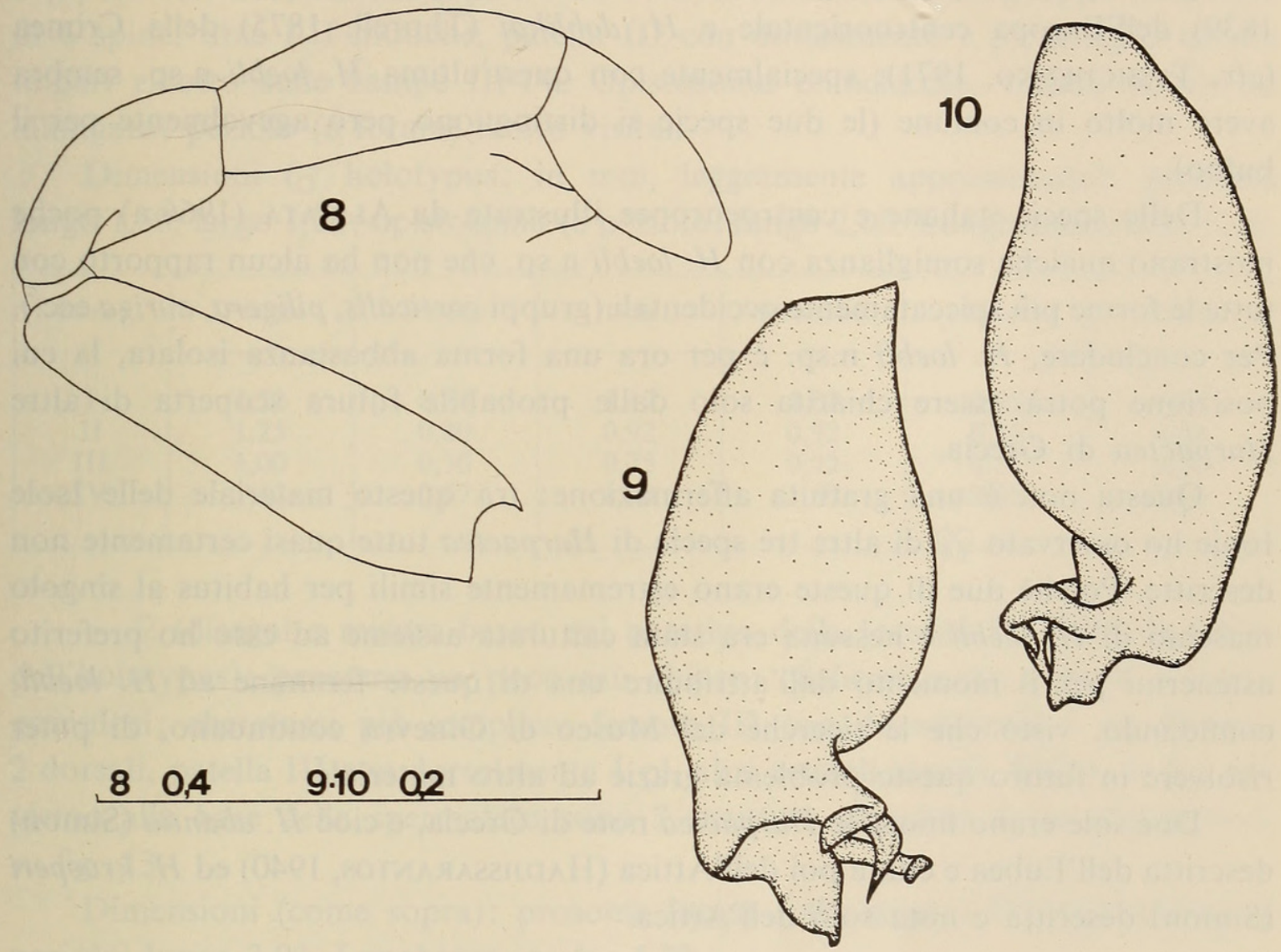

FIG. 8-10.

Harpactea loebli n. sp. - Fig. 8: palpo del ô; Fig. 9-10: bulbo dall'esterno e dall'interno.

(Nosek, 1905) (cfr. anche DrensKy, 1938 c) ha un bulbo leggermente simile, ma agevolmente distinguibile, da quello di $H$. loebli n.sp. Ben diverse dalla nuova specie sono anche le due uniche ad ampia diffusione, H. hombergi (Scopoli, 1763) e H. lepida (C. L. Koch, 1839). Se si estende il confronto agli Harpactocrates balcanici (che per lo più non hanno nulla a che fare con i « veri » Harpactocrates), $H$. storkani Kratochvil, 1935, non sembra avere nulla a che fare con questa linea filetica, mentre $H$. marani Kratochvil, 1937, può al massimo ricordare $H$. egregius (Kulczynski, 1897). Harpactea albanica (di Caporiacco, 1949), descritta contrariamente all'uso più frequente in questo gruppo sulla sola + , oltre ad essere grande il doppio di $H$. loebli n.sp. (lunga $7,5 \mathrm{~mm}$ ) ha i femori I con 4 spine $\mathrm{pl} \mathrm{e}$ le tibie II mutiche. 
Concludendo, di tutte le specie definibili come balcaniche, l'unica sotto certi aspetti simile a $H$. loebli n.sp. potrebbe essere $H$. sturanyi (Nosek) che però in realtà non è con certezza presente nella penisola balcanica, visto che la sua località tipica è il Serai Dag presso Konya in Turchia e che solo DrENSKY (1938 c) l'ha in seguito citata di Bulgaria.

Molto più somiglianti alla nuova specie sono invece $H$. rubicunda (C.L. Koch, 1839) dell'Europa centroorientale e $H$. doblikai (Thorell, 1875) della Crimea (cfr. TYSHCHENKO, 1971); specialmente con quest'ultima $H$. loebli n.sp. sembra avere molto in comune (le due specie si distinguono però agevolmente per il bulbo).

Delle specie italiane e centroeuropee illustrate da Alicata (1966 a) poche mostrano qualche somiglianza con $H$. loebli n.sp. che non ha alcun rapporto con tutte le forme più spiccatamente occidentali (gruppi corticalis, piligera, auriga ecc.). Per concludere, $H$. loebli n.sp. è per ora una forma abbastanza isolata, la cui posizione potrà essere chiarita solo dalla probabile futura scoperta di altre Harpactea di Grecia.

Questa non è una gratuita affermazione: tra questo materiale delle Isole Ionie ho osservato $q \circ$ di altre tre specie di Harpactea tutte quasi certamente non descritte. Poichè due di queste erano estremamente simili per habitus al singolo maschio di $H$. loebli e nessuna era stata catturata assieme ad esso ho preferito astenermi per il momento dall'attribuire una di queste femmine ad H. loebli, confidando, visto che le ricerche del Museo di Ginevra continuano, di poter risolvere in futuro questo problema grazie ad altro materiale.

Due sole erano finora le Harpactea note di Grecia, e cioè H. abantia (Simon) descritta dell'Eubea e citata poi dell'Attica (HADJISSARANTOS, 1940) ed H. krueperi (Simon) descritta e nota solo dell'Attica.

\section{Dasumia nativitatis $\mathrm{n}$. $\mathrm{sp}$.}

Morea - Massiccio del Panachaikon, strada da Patrasso a Kastrition, 650 m, 17.IV.72, B. Hauser leg., $1 \hat{\sigma}$ (Holotypus), 1 q (Paratypus), $2 \circ \circ$ (probabilmente conspecifici); Io-72/48).

Leucade - Presso la strada da Phryni a Tsoukalades, boschetto di ulivi, 14.IV.72,

V. Mahnert leg., 1 đ̃ (Paratypus; Io-72/38).

Corfù - Paleokastritsa, sotto ulivi presso il monastero, 75 m, 10.IV.72, B. Hauser

leg., 1 ô (Paratypus: Io-72/12).

Zante - Katastarion, macchia ai piedi delle rocce dietro il villaggio (vagliatura), 23.III.71, B. Hauser leg., 1 \% (Paratypus; Gr-71/7).

Descrizione - $\widehat{\jmath}$ : prosoma marrone, finemente granuloso, basso, a contorno angoloso; occhi in un gruppo compatto, ravvicinati, adeguali, posteriori 
in una linea a leggera concavità anteriore. Cheliceri bruni, anteriormente leggermente granulosi, con 2-1 denti (2 al margine inferiore). Palpo, v. fig. 11, 12, 14. Labium molto più lungo che largo; sterno ovale, gialliccio, appena granuloso, terminante con una piccola punta, separante le IV coxe molto meno del loro diametro. Chetotassi delle zampe (holotypus): femore I con 2 spine pl, II con $1 \mathrm{pl}$, III con dorsalmente $2 \mathrm{pl} \mathrm{e} 1 \mathrm{rl}$, IV con dorsalmente un gruppo irregolare di 6 spine, tibie I-II mutiche, patella III con dorsalmente $1 \mathrm{pl}$; artiglio tarsale impari ridotto sulle zampe III-IV. Opistosoma bianchiccio, villoso, sottile ad allungato; peziolo (e lorum) molto visibili.

Dimensioni ( $\hat{\sigma}$ holotypus; in $\mathrm{mm}$, leggermente approssimate): prosoma lungo 1,50, largo 1,22; opistosoma (e peziolo) lungo 2,00. Lunghezza totale: 3,50.

\begin{tabular}{|c|c|c|c|c|c|c|}
\hline Zampe & Femore & Patella & Tibia & Metatarso & Tarso & Totale \\
\hline I & 1,32 & 0,85 & 1,12 & 1,02 & 0,35 & 4,66 \\
II & 1,25 & 0,80 & 0,92 & 0,92 & 0,35 & 4,24 \\
III & 1,00 & 0,50 & 0,75 & 0,92 & 0,35 & 3,52 \\
IV & 1,42 & 0,67 & 1,20 & 1,50 & 0,35 & 5,14 \\
\hline
\end{tabular}

— $q$ (disegni e misure basati sul paratipo della località Io $72 / 48$, la stessa dell'holotypus): prosoma un poco più chiaro; cheliceri meno robusti e meno granulosi; chetotassi più semplice: femore III con dorsalmente $1 \mathrm{pl}$, IV con 2 dorsali, patella III con dorsalmente 1 pl, altri articoli mutici. Vulva, v. fig. 15; vicino alla base della spermateca sono 2 strutture singolari, forse ghiandolari, v. fig. 13 .

Dimensioni (come sopra): prosoma lungo 1,40, largo 1,12; opistosoma (e peziolo) lungo 3,00. Lunghezza totale: 4,40 .

\begin{tabular}{|c|c|c|c|c|c|c|}
\hline Zampe & Femore & Patella & Tibia & Metatarso & Tarso & Totale \\
\hline I & 1,00 & 0,67 & 0,82 & 0,75 & 0,30 & 3,54 \\
II & 0,95 & 0,60 & 0,77 & 0,70 & 0,30 & 3,32 \\
III & 0,80 & 0,45 & 0,55 & 0,75 & 0,30 & 2,85 \\
IV & 1,05 & 0,50 & 0,87 & 1,07 & 0,37 & 3,86 \\
\hline
\end{tabular}

Derivatio nominis: ho scoperto che questa specie era nuova il giorno di Natale, da qui il nome.

Affinità: ReImoser (1930 a) citò dell'isola di Leucade «Harpactes» amoenus Kulczynski, 1897 (ora Dasumia amoena), dato ripreso da BRISTOWE (1935 b), Hadjissarantos (1940) e RoEwer (1942), ma, stranamente, trascurato da Alicata (1966 b) il quale anzi (op. cit.: 480-81) insistette sull'assenza in 

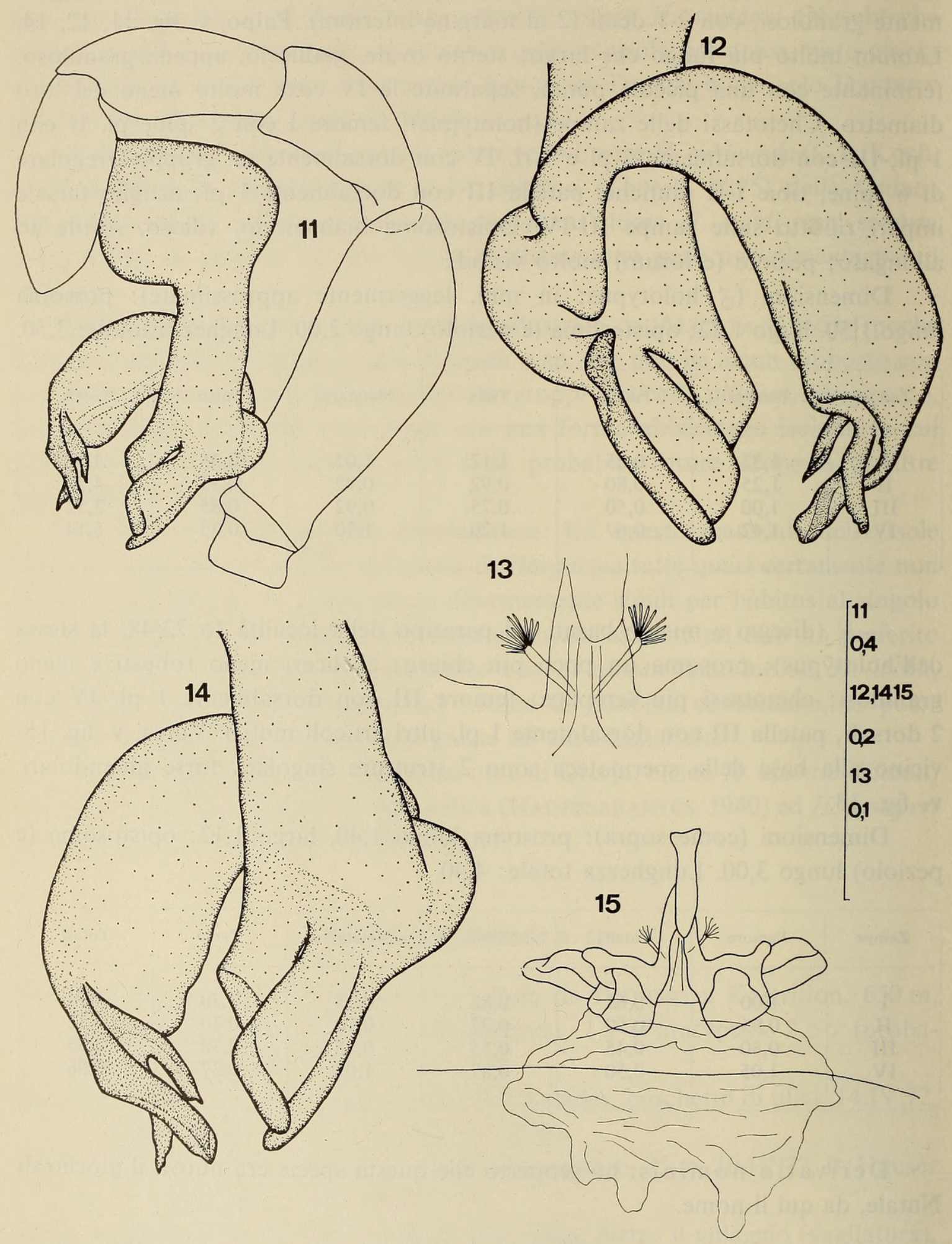

FIG. 11-15.

Dasumia nativitatis n. sp. - Fig. 11: palpo del §ै; Fig. 12, 14: embolo dall'esterno e dall'interno; Fig. 15: vulva; Fig. 13: strutture ghiandolari (?) annesse alla spermateca. 
Grecia ed in genere a Sud del solco transegeico del genere Dasumia vedendo in ciò una prova di una supposta origine nordegeica del genere. È altamente probabile quindi che l'«Harpactes amoenus» di ReIMOSER corrisponda a Dasumia nativitatis n.sp.

Lo studio di Alicata (op. cit.) sul genere Dasumia ha permesso l'immediata identificazione di questa specie come nuova; nessuna infatti ha un bulbo che si possa confondere con essa; $D$. amoena può in una certa misura ricordare la nuova specie (cfr. anche KULCZYNSKI, 1915), ma è chiaramente diversa. Oltre a $D$. amoena, mi sembrano affini a $D$. nativitatis n.sp. essenzialmente $D$. chyzeri (Kulczynski, 1906) della Dalmazia e D. diomedea di Caporiacco, 1948, delle Isole Tremiti, un gruppo di specie che mi pare abbastanza naturale. Il gruppo amoena-kusceri-carpathica proposto da AliCATA non mi pare molto omogeneo e specialmente D. kusceri (Kratochvil, 1935) mi sembra più vicina al gruppo laevigata-taeniifera-canestrinii, mentre più incerta è la posizione di $D$. carpathica (Kulczynski, 1882). Anche in questo genere però è probabile che manchino ancora degli «anelli di congiunzione » che potrebbero chiarire meglio le affinità; è per es. altamente verosimile che le citazioni di $D$. amoena della Bulgaria e della Crimea siano da ascrivere a specie differenti, probabilmente nuove. Sempre delle Isole Ionie ho tra l'altro delle ${ }_{+} \rightarrow$ appartenenti a quanto pare ad un'altra specie.

\section{Fam. LePtONETIDAE}

Sulcia violacea $\mathrm{n}$. sp.

Itaca - Campione di suolo sotto Cupressus presso Exoghi (selettore Berlese), 400 m, 19.IV.72, B. Hauser leg., 19 (Holotypus), $2 \circ 0$ (probabilmente conspecifici; Io-72/57).

Itaca - Grotta Nimfis presso Ithaki, 220 m, 19.IV.72, V. Mahnert leg., 1 ㅇ (Paratypus), 200 (probabilmente conspecifici; Io-72/85).

Descrizione - $q$ ( $\widehat{o}$ ignoto): prosoma marrone chiaro, piuttosto squadrato, specie anteriormente, occhi ben sviluppati, i due posteriori leggermente più grandi dei quattro anteriori $(6: 5)$, intervallo tra i posteriori ed i laterali del gruppo anteriore pari alla metà del diametro dei posteriori. Labium e gnatocoxe senza peculiarità; cheliceri, v. fig. 17; palpo, v. fig. 18; sterno bruniccio unicolore, cordiforme, ma con lati subparalleli (arieggiante un poco la condizione delle Segrea). Zampe color bruno chiaro. Opistosoma violaceo, più scuro ventralmente, sopra più chiaro con tre coppie di piccole macchie, posteriormente le macchie confluiscono in strisce trasversali decrescenti (N.B.: una colorazione così marcata è rarissima nei Leptonetidae e ricorda in modo sorprendente quella di molti Ochyroceratidae); vulva, v. fig. 19. 
Dimensioni (in $\mathrm{mm}$, leggermente approssimate): prosoma lungo 0,78 , largo 0,57 ; opistosoma lungo 1,37. Lunghezza totale: 2,15.

\begin{tabular}{|c|c|c|c|c|c|c|}
\hline Zampe & Femore & Patella & Tibia & Metatarso & Tarso & Totale \\
\hline I & 2,75 & 0,25 & 3,17 & 2,95 & 1,30 & 10,42 \\
II & 2,00 & 0,25 & 1,87 & 1,50 & 0,95 & 6,57 \\
III & 1,70 & 0,25 & 1,17 & 1,42 & 0,75 & 5,29 \\
IV & 2,12 & 0,25 & 2,15 & 1,87 & 1,00 & 7,39 \\
\hline
\end{tabular}

Derivatio nominis: "violacea» deriva dalla colorazione di questa specie, eccezionale per i Leptonetidae.

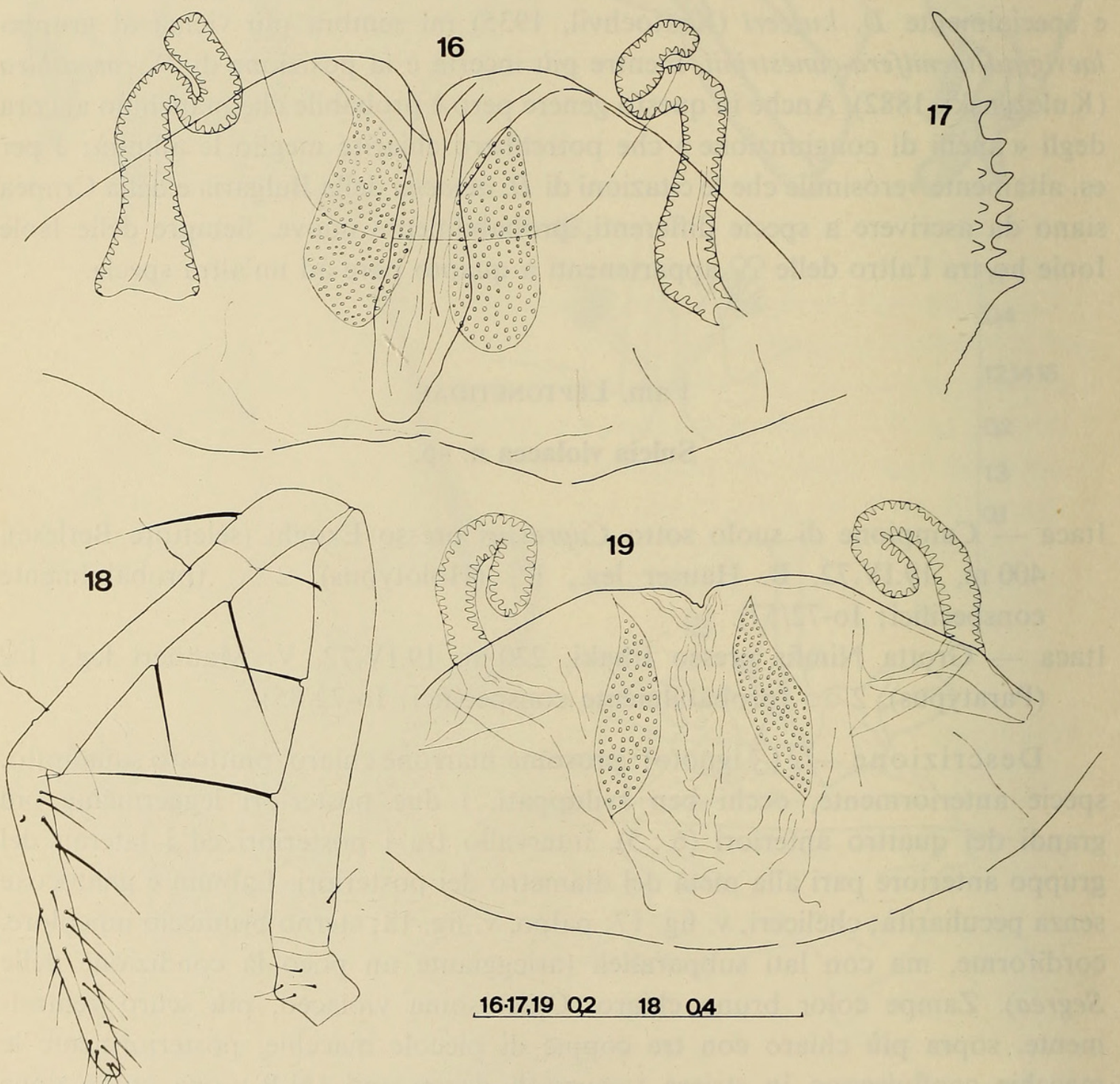

FIG. 16-19.

Sulcia violacea n. sp. - Fig. 17: margine del chelicero; fig. 18: palpo della +; Fig. 19: vulva. Sulcia cretica lindbergi Dresco, 1962 - Fig. 16: vulva (+ topotipica). 
Affinità: la nuova specie, in base alla morfologia generale ed alla vulva è prossima a Sulcia cretica lindbergi Dresco, 1962, della grotta di Perama nell'Epiro, che è anche la forma geograficamente più vicina. Per facilitare il confronto pubblico qui un nuovo disegno della vulva di quest'ultima forma (v. fig. 16); il disegno da me a suo tempo pubblicato $(1968$ b) è infatti incompleto. Anche nei Leptonetidae la tecnica tradizionale da me allora impiegata $(\mathrm{KOH}$ ed inclusione in Euparal) non permetteva grandi risultati, specie in breve tempo. La scoperta nelle Isole Ionie di una forma distinguibile, per morfologia dei genitali, dalla forma dell'Epiro pone dei nuovi, interessanti problemi riguardo alle Sulcia di

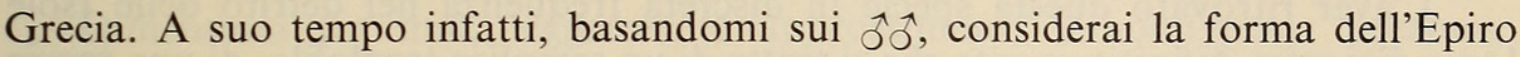
come razza della forma di Creta; non potei però esaminare $q 0$ della forma di Creta. È indubbiamente molto singolare che mentre in Epiro ed a Creta esistano Sulcia i cui $\widehat{\text { oิ }}$ s sono indistinguibili, nelle Isole Ionie esista una Sulcia prossima, ma distinguibile per i genitali femminili dalla forma dell'Epiro.

La soluzione di questi problemi è in parte possibile, visto che sono note †† della forma di Creta; c'è da augurarsi che in futuro vengano ritrovati ỗ $\widehat{o}$ di quella delle Isole Ionie. Le implicazioni più interessanti sono sul valore dei genitali come carattere tassonomico in questo gruppo, che potrebbe anche rivelarsi come un Rassenkreis analogo a quello delle Paraleptoneta tirreniche. Sulcia kanellisi Deeleman-Reinhold, 1971, dell'Attica (l'unica altra Sulcia di Grecia) non ha alcun rapporto con queste forme occidentali.

\section{Fam. THERIDIIDAE}

Pholcomma gibbum (Westring, 1851)

Morea - Patrasso, dintorni dell'università (campione di suolo), 21.III.71, B. Hauser leg., 1 ( $(\mathrm{Gr}-71 / 3)$.

Leucade - Bosco di ulivi sopra Kaligoni (vagliatura sotto arbusti in riva ad un fiume), 26.III.71, I. Löbl leg., 1 ô, 1 ㅇ (Gr-71/30).

Cefalonia - Margine della strada presso Livadion, 1.IV.71, B. Hauser leg., 1 ○ (Gr-71/64).

Specie nuova per la Grecia; in base anche a vari reperti inediti dell'Italia centromeridionale sembra molto più comune di quanto si poteva ritenere nell'Europa mediterranea.

Fam. Symphytognathidae

Pseudanapis apuliae di Caporiacco, 1949

Cefalonia - Sami, strada in direzione di Argostoli, a circa $11 \mathrm{~km}$ da Sami (vagliatura nella macchia, estrazione con apparecchio Berlese fino al 9.IV.), 6.IV.70, B. Hauser leg., 1 o (Ke-70/34). 
Cefalonia - Sami (vagliatura sotto Pistacia su di una collina a Sud del villaggio),

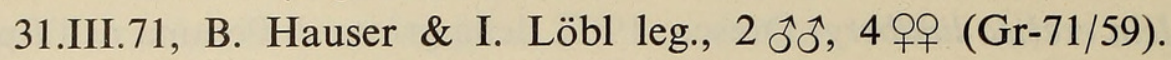

Leucade - Bosco di ulivi sopra Kaligoni (vagliatura sotto arbusti in riva ad un fiume), 26.III.71, I. Löbl leg., 1 $\widehat{o}$ (Gr-71/30).

Nuova per la Grecia; primi reperti in una stazione differente dalla località tipica (la grotta Zinzulusa nel Salento, Puglie). Il palpo dei $\widehat{o} \sigma \widehat{~(v . ~ f i g . ~ 20) ~ c o n-~}$ corda in maniera notevolissima con quello degli individui topotipici da me anni fa esaminati (BRIGNOLI, 1968 a).

L'aver ritrovato questa specie in discreta frequenza nel detrito delle Isole Ionie dimostra definitivamente che si tratta di un semplice troglofilo, di una specie quindi igrofila e termofila ormai ridotta a vivere in poche « isole » climaticamente adatte separate da zone aride. Voler vedere in specie con simili esigenze ecologiche relitti di epoca preglaciale o addirittura prepliocenica può essere attraente per zoogeografi troppo ossequienti alle vedute di Jeannel, ma è a mio parere prematuro. A parte infatti le fortissime alterazioni di origine antropica proprio nelle foreste e macchie mediterranee di bassa quota, è ben noto che nel glaciale ed anche nel postglaciale (cioè in epoca relativamente recente) vi sono stati parecchi periodi climaticamente adatti a specie con siffatte esigenze ecologiche (cfr. lo studio di BuTZER, 1958).

\section{Fam. HahniIdae}

Hahnia candida Simon, 1875

Morea - Patrasso, dintorni dell'università (da campione di suolo), 21.III.71, B. Hauser leg., 1 \% (Gr-71/3).

Nuova per la Grecia (nessun Hahniida era noto di questa regione).

\section{Hahnia ulyxis n. sp.}

Zante - Monte Skopos, m 330 circa (vagliatura sotto cipressi), 24.III.71, B. Hauser \& I. Löbl leg., 1 q (Paratypus), 1 o (Gr-71/15).

Zante - Presso Vasilikon, $100 \mathrm{~m}$ (vagliatura in una gola sotto Quercus), 22.IV.72, B. Hauser leg., 2 우 (Holo- et Paratypus; Io-72/67).

Descrizione - 우 ( $\hat{\jmath}$ ignoto): prosoma marrone olivastro chiaro, con setole nelle zone toracica ed oculare, fovea assente, solchi toracici appena evidenti, clipeo invisibile dall'alto; occhi anteriori in una linea a debole, posteriori in una linea a forte concavità anteriore; occhi simili per diametro (LA/ Posteriori : $\mathrm{MA}=4: 3$ ); anteriori equidistanti, assai ravvicinati (intervalli inferiori a metà del diametro dei MA), posteriori più distanziati (intervallo tra 
i MP appena inferiore al loro diametro, intervallo LP-MP pari a metà del loro diametro). Sterno unicolore olivastro, cordiforme, ampiamente troncato, separante le IV coxe del loro diametro, con molte setole lungo i margini. Zampe olivastre, metatarsi III-IV mutici, con solo una tricobotria nel terzo distale. Opistosoma inferiormente bianchiccio, dorsalmente con disegno assai simile a quello di $H$. picta Chyzer \& Kulczynski 1897 (cfr. HARM, 1966, fig. 57, pag. 366); stigma tracheale nettamente più vicino alle filiere che non al solco epigastrico; zona dell'epigino assai poco pigmentata (visibili per trasparenza i dotti), evidenti sono solo i due orifizi copulatori; vulva, v. fig. 21.

Dimensioni (in $\mathrm{mm}$, leggermente approssimate; $q$ holotypus): prosoma lungo 0,72 ; largo 0,56; opistosoma lungo 1,30. Lunghezza totale: 2,02.

\begin{tabular}{|c|c|c|c|c|c|c|}
\hline Zampe & Femore & Patella & Tibia & Metatarso & Tarso & Totale \\
\hline I & 0,50 & 0,16 & 0,33 & 0,25 & 0,25 & 1,49 \\
II & 0,50 & 0,16 & 0,27 & 0,25 & 0,22 & 1,40 \\
III & 0,38 & 0,16 & 0,22 & 0,25 & 0,20 & 1,21 \\
IV & 0,55 & 0,16 & 0,37 & 0,35 & 0,28 & 1,71 \\
\hline
\end{tabular}

Derivatio nominis: la nuova specie è dedicata all'omerico Ulisse, eroe così legato alle Isole Ionie.

Affinità: l'ottimo recente studio della HARM (1966) permette di distinguere agevolmente questa specie da quasi tutte quelle paleartico-occidentali per la morfologia della vulva; H. ulyxis n.sp. resta solo da confrontare con $H$. barbara Denis, 1937, dell'Algeria e $H$. insulana Schenkel, 1938 di Madera. Di quest'ultima specie è noto finora soltanto il $\hat{\jmath}$; in base alle figure ed alla descrizione di SCHENKEL (1938 a) questa specie ha un bulbo estremamente simile a quello di $H$. pusilla C. L. Koch, 1841, H. ononidum Simon, 1875, H. nava (Blackwall, 1841) e $H$. helveola Simon, 1875 . In altre parole, $H$. insulana può essere inserita senza difficoltà in un gruppo di specie assai omogeneo, non solo dal punto di vista dei genitali maschili, ma anche da quello dei genitali femminili (tutti con spermateche doppie o bursae assai sviluppate). Poichè $H$. ulyxis n.sp. ha dei genitali femminili di tutt'altra struttura, è logico supporre che anche i genitali maschili debbano essere di tipo diverso da quelli del gruppo pusilla. Altre differenze sono riscontrabili nella colorazione, nella lunghezza relativa delle zampe (I, II, IV, III in $H$. insulana) e nella chetotassi delle zampe (metatarsi III-IV con 1-1 spine, a parte il gruppo apicale, in $H$. insulana).

La descrizione di $H$. barbara (di cui è nota solo la ?) è corredata solo da una figurina, non troppo chiara, dell'epigino, con appena accennati i sottostanti genitali interni; dalle dimensioni, dalla colorazione e dall'epigino, nonchè dalle 
parole di DenIS ("very near to what I think to be H. muscicola Simon ", 1937 e: 1052) sembra trattarsi di una specie prossima a $H$. candida Simon, 1875 (= muscicola Simon), di un gruppo quindi ben distinto per morfologia dei genitali da H. ulyxis n.sp.

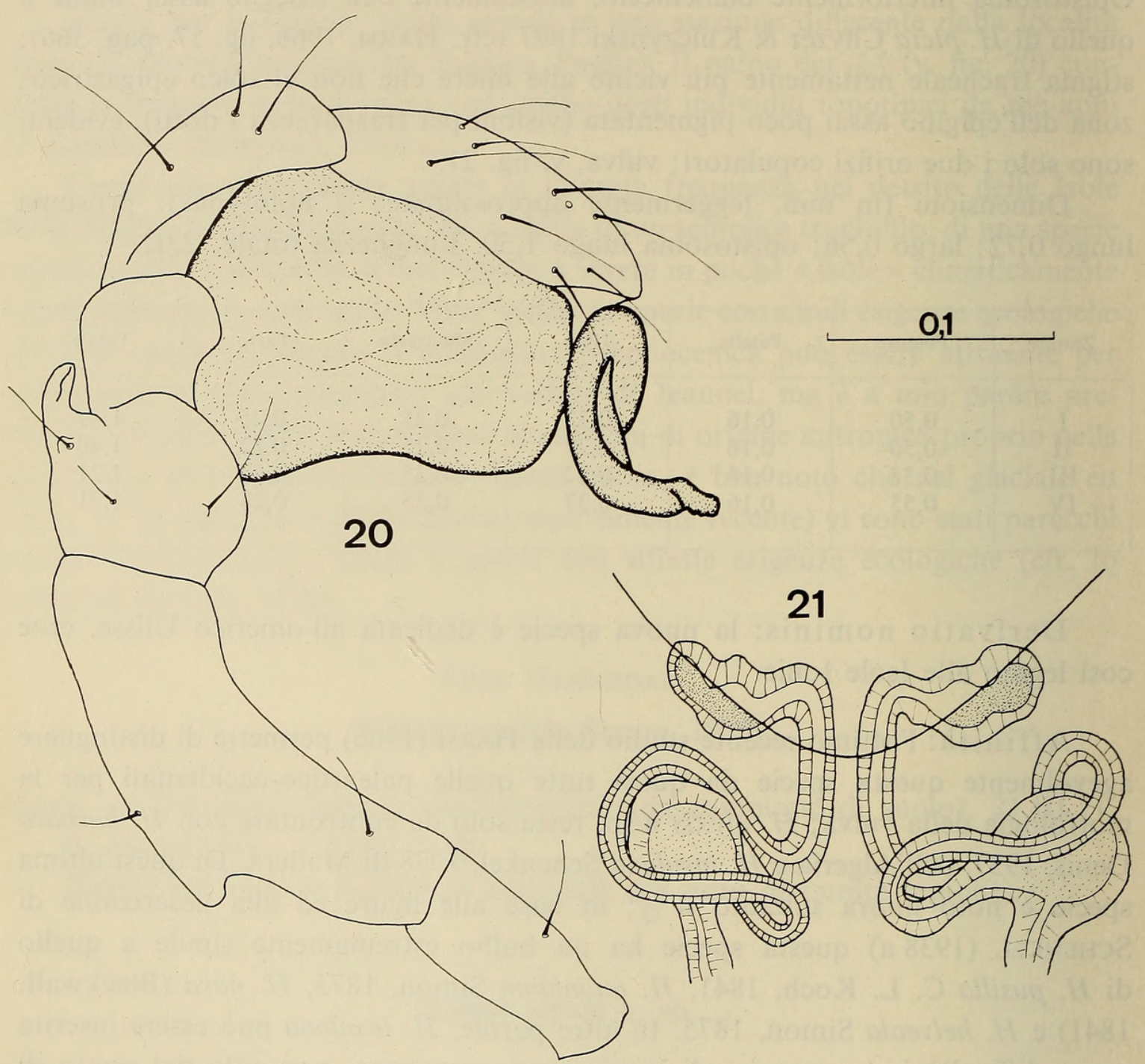

FIG. 20-21.

Pseudanapis apuliae di Caporiacco, 1949 - Fig. 20: palpo del ฮ̂.

Hahnia ulyxis n. sp. - Fig. 21: vulva.

La nuova specie, oltre che per ragioni geografiche, mi sembra essere affine anche per morfologia dei genitali solo a $H$. picta Chyzer \& Kulczynski, 1897 nota di Francia, Cecoslovacchia; Austria, Ungheria ed Italia (Puglie, reperto da controllare, cfr. Brignoli, 1973). In $H$. picta infatti il decorso dei dotti non è molto dissimile da quello di $H$. ulyxis n.sp. e gli orifizi di copulazione sono molti ampi, come nella nuova specie, nella quale però le spermateche sono ben evidenti. 


\section{SUMMARY}

In this paper a part of the results of the researches in the Ionian Islands and Peloponnesus (Greece) organized by the Muséum d'Histoire Naturelle de Genève is published. The following new species are described: Oonops mahnerti n.sp. (Oonopidae; + , $\hat{o}$ unknown; loc. typ.: Lefkas Island) of uncertain affinities, distinguishable by genitalia and/or chaetotaxy by the other known species; Folkia lugens n.sp. (Dysderidae; + , $\hat{o}$ unknown; loc. typ.: Lefkas Island) of uncertain affinities, distinguishable by the genitalia from the other known species; Harpactea loebli n.sp. (Dysderidae; $\hat{0}$, $q$ unknown; loc. typ.: Kephallenia Island) similar to $H$. doblikai (Thorell), H. rubicunda (C. L. Koch) and H. sturanyi (Nosek), distinguishable from these and the other known species by the genitalia; Dasumia nativitatis n.sp. (Dysderidae; $\hat{0}$ of loc. typ.: Peloponnesus, M. Panachaikon), near to D. chyzeri (Kulczynski) and D. diomedea di Caporiacco, distinguishable from these and the other known species by the genitalia; Sulcia violacea n.sp. (Leptonetidae; + , ô unknown; loc. typ.: Ithaca Island). near to $S$. cretica lindbergi Dresco, distinguishable from this species and the other known by the genitalia; Hahnia ulyxis n.sp. (Hahniidae; + , ô unknown; loc. typ.: Zakynthos Island), near to $H$. picta Chyzer \& Kulczynski, distinguishable from this species and the other known by the genitalia. The subgenus Folkia Kratochvil, 1970, is elevated to genus rank (generotypus: Stalagtia (Folkia) purkrabeki Kratochvil, 1970); Folkia is very near to Stalagtia Kratochvil, 1970 and Minotauria Kulczynski, 1903; these three genera can be distinguished by the morphology of the 0 -palpus. The Dysderidae of Creta attributed by Kratochvil (1970) to Stalagtia (Folkia) are transferred to Minotauria: $M$. cretica cretica (Roewer, 1928) comb. nov. and M. cretica fagei (Kratochvil, 1970) comb. nov.; both are very near to the generotype of Minotauria, M. attemsi Kulczynski, 1903. Orchestina simoni de Dalmas, 1916 (a 0 found at Lefkas) is new to Greece; a $q$ found at Ithaca is identical to a specimen from Sardinia determined by comparison with the type as O. setosa de Dalmas, 1916 by BRIGNOLI $(1967 \mathrm{~b})$; a possibility of a synonymy between $O$. simoni and $O$. setosa is supposed. New to Greece are also Pholcomma gibbum (Westring, 1851) (records of Peloponnesus, Lefkas and Kephallenia), Pseudanapis apuliae di Caporiacco, 1949 (records of Kephallenia and Lefkas; only other known stations), Hahnia candida Simon, 1875 (recorded from Peloponnesus). Some records of Dysderina loricatula Roewer, 1942 are listed.

\section{ZUSAMMENFASSUNG}

In dieser Arbeit wird ein Teil der Ergebnisse der Forschungen des Naturhistorischen Museums Genf auf den Ionischen Inseln und dem Peloponnes (Griechenland) veröffentlicht. Folgende neue Arten werden beschrieben: Oonops 
mahnerti n.sp. (Oonopidae; nach einem + ; das $\hat{0}$ ist unbekannt; locus typicus: die Insel Levkas) von unsicherer Verwandtschaft, von den übrigen bisher bekannten Arten durch die Genitalien und (oder) die Chaetotaxie unterscheidbar; Folkia lugens n.sp. (Dysderidae; nach einem + ; das $\widehat{\jmath}$ unbekannt; locus typicus: die Insel Levkas) von unsicherer Verwandtschaft, durch die Genitalien von den übrigen bisher bekannten Arten unterscheidbar; Harpactea loebli n.sp. (Dysderidae; nach einem $\hat{o}$; das + unbekannt; locus typicus: die Insel Kephallinia) ähnlich H. doblikai (Thorell), H. rubicunda (C. L. Koch) und H. sturanyi (Nosek), von diesen und den übrigen bisher bekannten Arten durch die Genitalien unterscheidbar; Dasumia nativitatis n.sp. (Dysderidae; nach $\widehat{\jmath}$ und ; locus typicus: Peloponnes, Panachaikon-Gebirge), nahestehend D. chyzeri (Kulczynski) und D. diomedea di Caporiacco, von diesen und den übrigen bisher bekannten Arten durch die Genitalien unterscheidbar; Sulcia violacea n.sp. (Leptonetidae; nach einem ; das $\widehat{\jmath}$ unbekannt; locus typicus: die Insel Ithaka), nahestehend S. cretica lindbergi Dresco, von dieser und den übrigen bisher bekannten Arten durch die

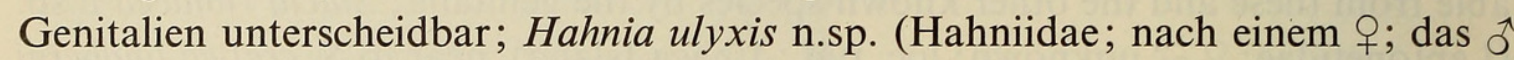
unbekannt; locus typicus: die Insel Zakynthos), nahestehend $H$. picta Chyzer et Kulczynski, von dieser und den übrigen bisher bekannten Arten durch die Genitalien unterscheidbar. Die Untergattung Folkia Kratochvil, 1970 wird in den Rang einer Gattung erhoben (Gattungstypus: Stalagtia (Folkia) purkrabeki Kratochvil, 1970); Folkia steht sehr nahe Stalagtia Kratochvil, 1970 und Minotauria Kulczynski, 1903; diese drei Gattungen können durch die Morphologie des $\hat{0}$-Palpus unterschieden werden. Die Dysderidae Kretas von KrATOCHVIL (1970) zu Stalagtia (Folkia) gestellt, werden der Gattung Minotauria zugestellt: M. cretica cretica (Roewer, 1928) comb.nov. und $M$. cretica fagei (Kratochvil, 1970) comb.nov.; beide stehen der Typusart der Gattung. M. attemsi Kulczynski, 1903, sehr nahe. Orchestina simoni de Dalmas, 1916 (ein $\widehat{0}$ wurde auf der Insel Levkas gefunden) ist neu für Griechenland; ein $ᄋ$, das auf der Insel Ithaka gefunden wurde, ist identisch mit einem Exemplar von Sardinien, das von BRIGNOLI (1967 b) durch Typenvergleich als $O$. setosa de Dalmas, 1916 determiniert worden war; die Möglichkeit einer Synonymie zwischen $O$. simoni und O. setosa wird angenommen. Neu für Griechenland sind auch Pholcomma gibbum (Westring), 1851 (Nachweise vom Peloponnes, den Inseln Levkas und Kephallinia). Pseudanapis apuliae di Caporiacco, 1949 (Nachweise von Kephallinia und Levkas, die ersten Funde ausserhalb des locus typicus), Hahnia candida Simon, 1875 (vom Peloponnes nachgewiesen). Einige Nachweise von Dysderina loricatula Roewer, 1942 werden angegeben. 


\section{BIBLIOGRAFIA}

NB.: Per i lavori anteriori al 1939 rimando alla "Bibliographia Araneorum » di P. BONNET.

Alicata, P. 1966a. Le Harpactea della fauna italiana e considerazioni sulla loro origine. Atti Accad. gioenia Sci. nat. (6) 18: 190-221.

AlicatA, P. 1966 b. Il genere Dasumia Thorell, sua nuova definizione e revisione delle specie italiane. Memorie Mus. civ. Stor. nat. Verona 14: 465-486.

Brignoli, P. M. 1967a. Su alcuni Oonopidae delle Isole Ponziane. Fragm. ent. 4: 141-148.

Brignoli, P. M. 1967b. Oonopidae di Sardegna e Puglie. Rc. Ist. lomb. Sci. Lett. (B) 101: 360-368.

Brignoli, P. M. 1968a. Über zwei italienische Pseudanapis-Arten. Senckenberg. biol. 49: 131-136.

BRIGNOLI, P. M. 1968b. Über griechische Leptonetidae. Senckenberg. biol. 49: 259-264.

BRIGNoli, P. M. 1972. Eine neue Orchestina aus Griechenland. Senckenberg. biol. 53: 287-289.

Brignol, P. M. 1973. Ragni d'Italia .XX. Note sugli Hahniidae. Fragm. ent. 8: 265-274.

Butzer, K. W. 1958. Quaternary stratigraphy and climate in the Near East. Bonn. Geogr. Abh. 24: 1-157.

Caporiacco, L. di 1949. Alcuni aracnidi albanesi. Atti Mus. civ. Stor. nat. Trieste 17: $122-125$.

Deeleman- Reinhold, C. L. 1971. A new species of Sulcia Kratochvil from Greece, and a discussion of some Japanese cavernicolous Leptonetidae. Zool. Meded. Leiden 45: 289-301.

FAGE, L. 1943. Description d'une Leptonète de Corse suivie de remarques sur les araignées cavernicoles du genre Stalita. Bull. Mus. natn. Hist. nat. Paris 15:171-174.

FAGE, L. 1945. A propos de quelques araignées cavernicoles de Crète. Bull. Mus. natn. Hist. nat. Paris 17: 109-114.

Hadjissarantos, H. 1940. Les araignées de l'Attique. Athènes, 132 pp.

HARM, M. 1966. Die deutschen Hahniidae Senckenberg. biol. 47: 345-270.

Kratochvil, J. 1970. Cavernicole Dysderae. Prírodov. Pr. Ce'sk. Akad. H. N. S. 4: 1-62.

Roewer, C. F. 1942. Katalog der Araneae von 1758 bis 1941). Bremen, 1040 pp.

TYSHCHEnko, V. P. 1971. Opredelitel' paukov evropejskoj casti SSSR. Leningrad, 281 pp.

Adresse de l'auteur :

Istituto di Zoologia

Viale dell'Universita 32

I-00100 Roma

Italie 


\section{$2 \mathrm{BHL}$ Biodiversity Heritage Library}

Brignoli, Paolo Marcello. 1974. "Ragni di Grecia VI. Specie nuove interessanti delle isole Ionie e della Morea (Araneae)." Revue suisse de zoologie 81, 155-175. https://doi.org/10.5962/bhl.part.75999.

View This Item Online: https://www.biodiversitylibrary.org/item/126812

DOI: https://doi.org/10.5962/bhl.part.75999

Permalink: https://www.biodiversitylibrary.org/partpdf/75999

\section{Holding Institution}

Smithsonian Libraries

\section{Sponsored by}

Biodiversity Heritage Library

\section{Copyright \& Reuse}

Copyright Status: In Copyright. Digitized with the permission of the rights holder.

Rights Holder: Muséum d'histoire naturelle - Ville de Genève License: http://creativecommons.org/licenses/by-nc-sa/3.0/

Rights: https://www.biodiversitylibrary.org/permissions/

This document was created from content at the Biodiversity Heritage Library, the world's largest open access digital library for biodiversity literature and archives. Visit BHL at https://www.biodiversitylibrary.org. 